\title{
Integrated Method of Assessment the Type of Damages, their Localization and Predicting the Destruction of High-Voltage Electrical Equipment Insulators by the Partial Discharges Characteristics
}

\author{
Gataullin A.M., Gubaev D.F. \\ Kazan State Power Engineering University \\ Kazan, Russian Federation
}

\begin{abstract}
The aim of this study is to improve the accuracy of localization of defects in insulators and determine their type. This goal is achieved by solving the problem of combining contact and remote methods for polymer and porcelain insulators using model partial discharges. The most significant results are the regularities of the dynamics of the characteristics of partial discharges up to the prebreakdown situation for porcelain insulators, the study of the statistical distributions of partial discharges depending on their intensity, and the identification of the features of the statistical distribution of surface discharges. Part of the work is devoted to the study of the characteristics of partial discharges and their sources by spectra, polarity, statistical distributions, oscillograms, which is important from the point of view of automating the recognition of corona and internal partial discharges, as well as for the recognition of porcelain insulators destroyed by partial discharges. Regularities of changes in the statistical distribution of partial discharges up to the pre-breakdown situation were established. At the same time, the breakdown signs of the model discharge gap, the breakdown voltage values for defective and operable porcelain insulators are determined, which can be used to train models of artificial neural networks and recognize the pre-breakdown situation based on them. The most significant results were: assessment of the ohmic resistance of porcelain insulators by the characteristics of partial discharges, recognition of corona, internal and surface partial discharges of polymer insulators, localization of defects, using electromagnetic radiation sensors.
\end{abstract}

Keywords: partial discharges method, diagnostics of high-voltage electrical equipment insulators, localization of high-voltage insulators defects, state of high-voltage electrical equipment monitoring.

DOI: https://doi.org/10.52254/1857-0070.2022.1-53.12

UDC: 621.3.048.1

Metodă complexă de evaluare a caracterului deteriorării, localizare și predicție a defectării izolatorului în echipamentele electrice de înaltă tensiune pe baza caracteristicilor descărcărilor parțiale

Gataullin A.M., Gubaev D.F.

Universitatea de Stat de Energetică din Cazan, Federația Rusă, Kazan

Rezumat. Scopul cercetării este de a îmbunătăți precizia localizării defectelor în izolație și determinarea tipului defectelor. Obiectivul este atins prin rezolvarea problemei de combinare a metodelor de contact și la distanță pentru izolatori din polimer și porțelan folosind descărcările parțiale (DP) model. Cele mai semnificative rezultate sunt legitățile obținute ale dinamicii caracteristicilor DP până la situația de pre-străpungere pentru izolatorii de porțelan, investigarea distribuției statistice a DP în funcție de intensitatea lor și identificarea particularităților distribuției statistice a descărcărilor de suprafață. O parte din lucrare este dedicată studierii caracteristicilor DP și a surselor lor după spectre, polaritate, distribuții statistice, oscilograme, importanța fiind din considerente de automatizare la depistarea DP interioare și de tipul arcului electric, de asemenea, pentru depistarea izolatoarelor din porțelan deteriorate de DP. Astfel, au fost determinate: semnele de defecțiune ale unui decalaj model semnalmentele de străpungere a intervalului de descărcare model, valoarea tensiunii de străpungere pentru izolatorul de porțelan defectat și cel funcțional, care pot fi utilizate pentru învățarea modelelor de rețele neuronale artificiale și pentru a depista în baza acestora anterioară defectării pe baza acestora. Relevanța rezultatelor constă în faptul, că în baza caracteristicilor DP se identifică izolatorii din polimeri, fiind umectați umeziți, inclusiv printre izolatorii de porțelan umectați. Cele mai semnificative rezultate sunt: evaluarea rezistenței ohmice a izolatorilor de porțelan în baza caracteristicilor DP, depistarea DP de tipul arc electric, interioare și de suprafață ale izolatorilor din polimeri, localizarea defectelor folosind senzori de radiaţie electromagnetică din benzile UHF și HF.

Cuvinte-cheie: metoda descărcării parțiale, diagnosticarea izolatorilor echipamentelor electrice de înaltă tensiune, localizarea defectelor în izolatorii de înaltă tensiune, monitorizarea stării echipamentelor electrice de înaltă tensiune. 


\section{Комплексный метод оценки характера повреждений, их локализации и прогнозирования разрушения изоляторов высоковольтного электрооборудования по характеристикам частичных разрядов \\ Гатауллин А.М., Губаев Д.Ф.}

Казанский Государственный Энергетический Университет, Казань, Российская Федерация

Аннотация. Целью исследования является повышение точности локализации дефектов изоляторов и определение их типа. Поставленная цель достигается путем решения для полимерных и фарфоровых изоляторов задачи комбинирования контактного и дистанционного методов при использовании модельных частичных разрядов. Наиболее существенными результатами являются полученные закономерности динамики характеристик частичных разрядов вплоть до предпробойной ситуации для фарфоровых изоляторов, исследование статистических распределений частичных разрядов в зависимости от их интенсивности и выявление особенностей статистического распределения поверхностных разрядов. Часть работы посвящена изучению характеристик частичных разрядов и их источников по спектрам, полярности, статистическим распределениям, осциллограммам, что важно с точки зрения автоматизации распознавания коронных и внутренних частичных разрядов, а также для распознавания разрушенных частичными разрядами фарфоровых изоляторов. В работе приведены результаты экспериментов, в ходе которых сопоставлены результаты экспериментов для дефектных и бездефектных фарфоровых изоляторов и впервые установлено, что метод частичных разрядов с высокой степенью точности коррелирует с результатами, полученными методом прямого измерения омического сопротивления. Установлены закономерности изменения статистического распределения частичных разрядов вплоть до предпробойной ситуации. При этом определены: признаки пробоя модельного разрядного промежутка, значения пробивного напряжения для дефектного и работоспособного фарфорового изоляторов, которые могут быть применены для обучения моделей искусственных нейронных сетей и распознавания на их основе предпробойной ситуации. Значимость результатов состоит в том, что по характеристикам частичных разрядов выявляются увлажненные полимерные изоляторы, в том числе среди увлажненных фарфоровых изоляторов. Наиболее значимыми результатами являются: оценка по характеристикам частичных разрядов омического сопротивления фарфоровых изоляторов, распознавание коронных, внутренних и поверхностных частичных разрядов полимерных изоляторов, локализация дефектов с применением датчиков УВЧ и ВЧ диапазона электромагнитного излучения.

Ключевые слова: метод частичных разрядов, диагностика изоляторов высоковольтного электрооборудования, локализация дефектов высоковольтных изоляторов, мониторинг состояния высоковольтного электрооборудования.

\section{ВВЕДЕНИЕ}

\section{Описание проблемы}

В настоящее время значительное внимание уделяется развитию диагностики изоляторов высоковольтного электрооборудования по характеристикам частичных разрядов (ЧР). Известно, что с одной стороны, ЧР являются причиной разрушения высоковольтных изоляторов, а с другой стороны, по их характеристикам можно оценить состояние изоляторов и предсказать их разрушение. При этом единственными эффективными методами, применяемыми на практике, являются метод мегаомметра и метод повышенного пробивного напряжения, а критерием выявления дефектных изоляторов является критическое значение омического сопротивления.

До настоящего времени не разработано надежных и точных критериев выявления фарфоровых изоляторов с развитой системой внутренних дефектов в форме воздушных вкраплений (каверн) и загрязненных полимерных изоляторов, основанных на анализе характеристик ЧР, которые могут дать информацию о степени разрушения изоляторов, перекрытия разрядных промежутков. Решению задачи повышения точности диагностики изоляторов посвящено большое количество международных публикаций, связанные, в частности, с попыткой кластеризацией типов частичных разрядов и соответствующих им дефектов изоляторов с учетом особенностей распределения частичных разрядов по фазе приложенного напряжения, особенностей их спектров и форм сигналов. При этом, несмотря на достаточно большое количество исследований, в том числе с применением искусственных нейронных сетей, проблемы распознавания типов дефектов, например, внутренние дефекты, или поверхностное загрязнение, проблема локализации дефектов, особенно при наличии нескольких источников ЧР, a также проблема распознавания предпробойной ситуации разрядного промежутка до сих пор окончательно не 
решены. Также не разработаны методы прогнозирования пробоя для модельного разрядного промежутка под воздействием переменного приложенного напряжения, методы оценки омического сопротивления и пробивного напряжения для фарфоровых и полимерных изоляторов.

\section{Обзор литературы и описание нерешенных проблем}

В ряде работ [1-2] искусственная нейронная сеть (ИНР) обучалась распознаванию внутренних ЧР, поверхностных ЧР (ПЧР) и коронных разрядов, источником которых была система поверхность-игла. При этом ИНР обучали на основе осциллограмм ЧР и распределений ЧР по фазе приложенного напряжения, так называемых амплитудно-фазовых диаграммам (АФД). Недостатком этих работ является то, что в них не рассмотрены способы обучения ИНР для локализации источника ЧР. Ряд работ посвящены классификации типов ЧР по их осциллограммам и АФД [4-6], однако при этом не было изучено влияние фактора увлажнения поверхности изоляционных конструкций. Часть работ посвящена исследованию особенностей ЧР на подстанциях с элегазовой изоляцией, при этом исследования проводились на модельных источниках ЧР. Например, в работе [7], выполненной с применением УВЧ датчика ЧР, учитываются ЧР не только в элегазе, но и ПЧР, обусловленные загрязнением поверхности твердых изоляторов элегазовой подстанции. Однако в этой работе не рассмотрены особенности загрязнения поверхности фарфоровых и полимерных изоляторов и способы их разделения по характеристикам ЧР. В работе [8] рассматриваются дефекты внутри муфтовых соединений кабелей с изоляцией из сшитого полиэтилена. При этом были созданы искусственные внутренние дефекты. Среди них выделим дефекты трех типов: обусловленные наличием каверн внутри сшитого полиэтилена, дефекты, обусловленные появлением металлических опилок внутри муфты кабеля на поверхности изоляции из сшитого полиэтилена, и дефект, обусловленный некачественным монтажом кабеля, в виде торчащего куска проволоки жилы кабеля. В данной работе перечисленные дефекты хорошо распознаются по АФД, однако, ПЧР концевых муфт кабелей не рассматриваются. Многие авторы для увеличения соотношения сигнал/шум и выделения внутренних ЧР применяют датчики УВЧ диапазона электромагнитного излучения (ЭМИ) [9-10]. УВЧ датчики более предпочтительны с точки зрения локализации дефекта в силовых трансформаторах [11-13]. Иногда УВЧ датчики комбинируют с датчиками оптического диапазона ЭМИ [14]. Однако применение датчиков оптического диапазона позволяет лишь фиксировать ЧР, причем вблизи их возникновения и не позволяет подробно анализировать такие характеристики ЧР как, например, полярность импульсов, что важно с точки зрения распознавания типов ЧР и локализации дефектов. Для локализации дефекта на значительном расстоянии перспективно совместное применение датчиков ВЧ и УВЧ диапазонов ЭМИ, которые с этой точки зрения имеют ряд преимуществ, в том числе по сравнению с акустическими методами [15-17]. При этом ПЧР необходимо отделять от внутренних ЧР, которые разрушают изолятор изнутри [18-20].

\section{Цель исследования}

Целью исследования является увеличение точности локализации источника ЧР, прогнозирование предпробойной ситуации разрядного промежутка модельных ЧР, распознавание внутренних ЧР фарфоровых изоляторов на фоне ПЧР полимерных изоляторов, оценка омического сопротивления и пробивного напряжения дефектных фарфоровых изоляторов по характеристикам ЧР за счет комбинирования регистрации ЧР в ВЧ и УВЧ диапазонах ЭМИ, сопоставления характеристик ЧР и результатов прямого измерения омического сопротивления изоляторов.

\section{II. МЕТОДЫ ИССЛЕДОВАНИЯ \\ Описание установки}

Для решения поставленных в работе целей была собрана лабораторная установка, состоящая из высоковольтного источника переменного напряжения частотой 50 Гц и постоянного напряжения, источника модельных разрядов поверхность-игла, полимерного, фарфорового изоляторов, датчика ВЧ диапазона ЭМИ, широкополосной полуволновой антенны и УВЧ антенны (рис. 1). Для визуализации сигнала электромагнитной активности полуволновая антенна была оснащена $\mathrm{RC}$-цепочкой 
(постоянная времени 14 мкс), также применялся осциллограф Tektronix TDS-1012 с интерфейсом RS-232 и персональный компьютер с программой OpenChoice, плата сбора данных NI PCI-6251 с частотой дискретизации 1 МГц и программный анализатор сигнала реализованный в

\section{Условия возникновения ЧР}

Известно, что основной причиной разрушения твердой изоляции являются, прежде всего, внутренние ЧР. Условием возникновения внутренних ЧР в твердой изоляции является наличии в объеме изоляции воздушных вкраплений (каверн), которые

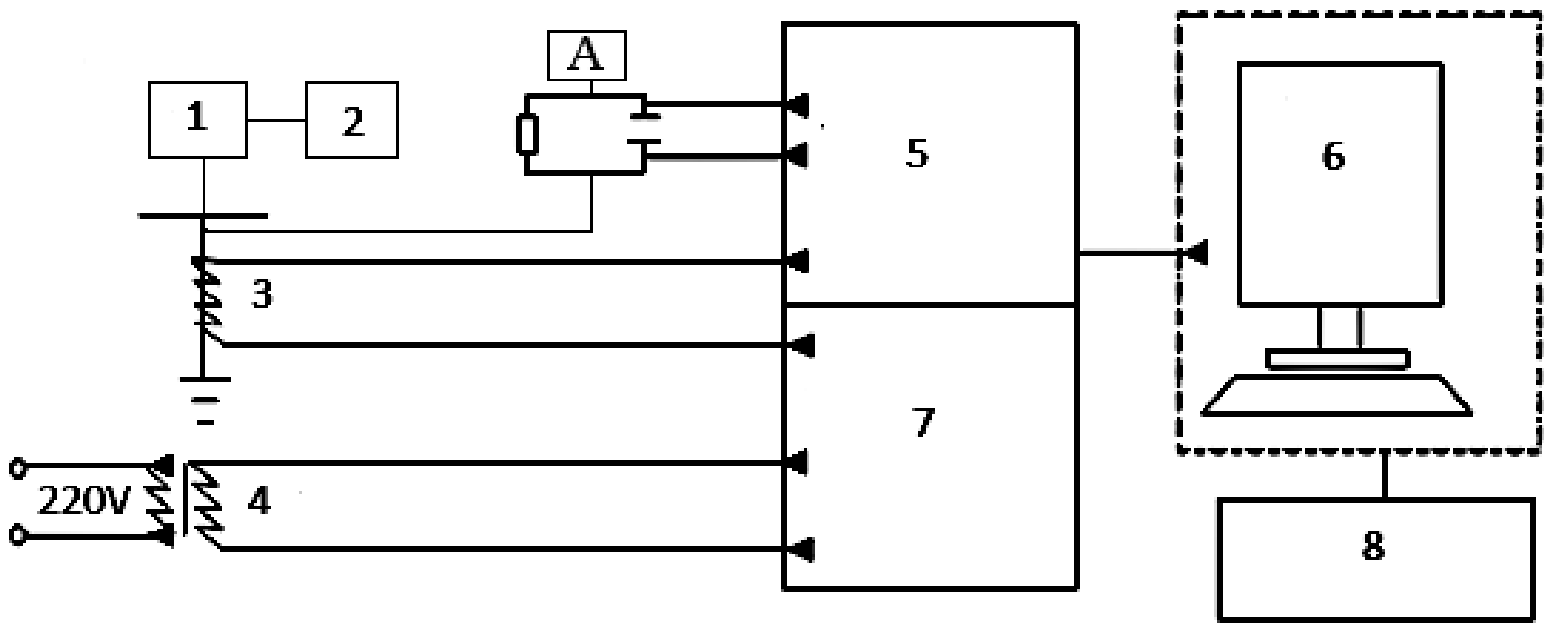

Рис. 1. Схема измерения. 1 - объект исследования (система поверхность-игла, полимерный изолятор, фарфоровый изолятор), 2 - блок высокого напряжения, 3 - высокочастотный измерительный импульсный трансформатор, 4 - трансформатор опорного напряжения, А - полуволновая широкополосная антенна и УВЧ антенны, 5 - плата аналого-цифрового преобразователя National Instruments, 6 - персональный компьютер, 7 - осциллограф Tektronix TDS 1012, 8 - программное обеспечение осциллографа Tektronix TDS 1012 и платы NI.

Fig. 1. Measurement scheme. 1 - the object of study (surface-needle system, polymer insulator, porcelain insulator), 2 - high voltage unit, 3 - high-frequency measuring pulse transformer, 4 - reference voltage transformer, A - half-wave broadband antenna and UHF antennas, 5 - National Instruments analog-to-digital converter board, 6 - is a personal computer, 7 - Tektronix TDS 1012 oscilloscope, 8 - software of the Tektronix

TDS 1012 oscilloscope and NI boards.

LabVIEW. Полоса пропускания осциллографа Tektronix TDS 1012 равна 100 МГц, максимальная частота дискретизации 1 ГГц, что позволяет регистрировать ЧР с длительностью фронта порядка наносекунд.

В работе были исследованы характеристики ЧР системы поверхностьигла, бездефектного и дефектного фарфорового подвесного изоляторов тарельчатого типа, а также ПЧР бездефектного полимерного изолятора. Параметры разрядной ячейки: расстояние от плоскости до иглы - 15 мм, диаметр иглы - 30 мкм, форма наконечника иглы плоская. Трансформатор опорного напряжения применялся для построения АФД. Для обработки сигналов на ПК применилось специальное программное обеспечение для осциллографа и платы NI. наряду с другими физическими и химическими неоднородностями изоляции способствуют ее разрушению. Каверны образуются на этапе производства и монтажа изоляторов. Известно, что возникновение ЧР в каверне происходит за счет образования резко неоднородного электрического поля. ЧР постепенно увеличивают число каверн. Условием возникновения ЧР в воздухе при нормальных условиях является соотношение:

$$
E_{l o c} / n \geq 90 T d
$$

где $E_{l o c}-$ напряженность локального электрического поля [B/M], $n$ - концентрация молекул $\left[\mathrm{M}^{-3}\right], \mathrm{Td}$ (таунсенд) - единица измерения соотношения $E_{l o c} / n$, $[1 \mathrm{Td}]=\left[10^{-21} \mathrm{BM}^{2}\right]$.

При условии выполнения соотношения (1) возникают внутренние ЧР, постепенно появляются новые каверны, а при достижении 
числа каверн критического значения, изоляция разрушается и происходит пробой. Дефект имеет собственную электрическую емкость, на которой периодически происходит частичный пробой, при условии превышения локального электрического поля критического значения (рис. 2). Амплитуда ЧР связана со степенью неоднородности напряженности электрического поля соотношением величин напряженности локального и среднего электрического поля

$$
E_{l o c} / E,
$$

где $E_{l o c}-$ напряженность локального электрического поля $[\mathrm{B} / \mathrm{M}], E-$ среднее значение напряженности электрического поля [B/м]. Относительная величина локального электрического поля определяется геометрией каверны, величиной остаточного заряда, влагой и другими параметрами. Схема замещения дефекта в диэлектрике представлена на рис. 2 .

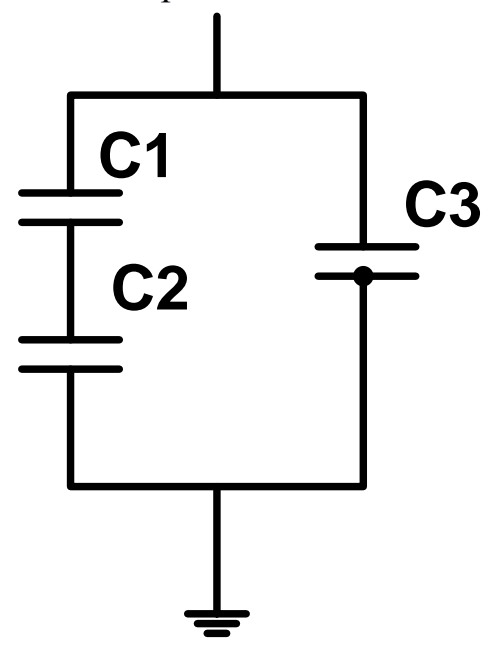

Рис. 2. Схема замещения дефекта в диэлектрике. $C_{1}-$ электрическая емкость неповрежденной изоляции, $C_{2}$ - электрическая емкость, обусловленная воздушной каверной, $C_{3}-$ электрическая емкость неповрежденной части изоляции.

Fig. 2. The equivalent electrical circuit of the defect in the dielectric. $C_{1}$ - electrical capacity of the intact insulation, $C_{2}-$ electrical capacity due to the air cavity $C_{3}$ - electric capacitance of intact insulation.

$$
\begin{aligned}
& q=C_{2}\left(V_{i}-V_{d}\right), \\
& C_{2} \gg C_{1},
\end{aligned}
$$

где $q-$ амплитуда ЧР $[n K \pi], \quad C_{2}-$ электрическая емкость изоляции $[H \Phi], C_{1}-$ электрическая емкость изоляции $[H \Phi], V_{i}-$ напряжение зажигания ЧР $[\mathrm{M} B], V_{d}-$ напряжение гашения ЧР [M $B]$.

Чем больше число дефектов одинакового типа находятся в один и тот момент времени под одинаковым потенциалом электрического поля, тем больше одновременно будут возникать ЧР $\mathrm{c}$ одной и той величиной кажущегося заряда.

\section{II. ОБСУЖДЕНИЕ РЕЗУЛЬТАТОВ}

Наибольший интерес представляли ЧР при переменном напряжении в предпробойной ситуации (рис. 3), которые сопоставлялись с ЧР при постоянном приложенном напряжении (импульсами Тричела) (рис. 4). При этом отдельно рассматривались ЧР положительной полярности и отрицательной полярности, причем ЧР отрицательной полярности интерпретировались как внутренние ЧР. Увеличение частоты следования ЧР отрицательной полярности, упорядоченных в «пачки», так называемые наиболее характерные ЧР, свидетельствовали о приближении к предпробойной ситуации по аналогии с импульсами Тричела, возникающие при постоянном приложенном напряжении.

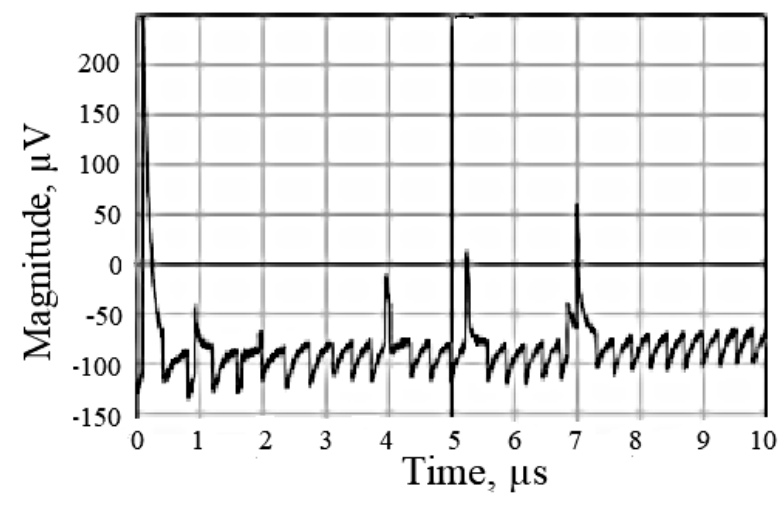

Рис. 3. Сигнал с антенны. Развернутый сигнал на отрицательной полуволне действующего напряжения. Предпробойная ситуация. Переменное напряжение 18 кВ. ЧР на фоне модулированного сигнала действующего напряжения появляются «пачками», ЧР в «пачках» упорядочены (наиболее характерные ЧР).

Fig. 3. Signal from the antenna. An expanded signal on the negative half-wave of the alternating applied voltage $18 \mathrm{kV}$. Modulated PDs appears one by one (the most typical PDs). 
Частота следования импульсов Тричела, как и ожидалось, росла с увеличением амплитуды приложенного напряжения, затем происходил пробой разрядного промежутка.

Похожая закономерность была обнаружена и для наиболее характерных ЧР, возникающих под воздействием переменного приложенного напряжения.

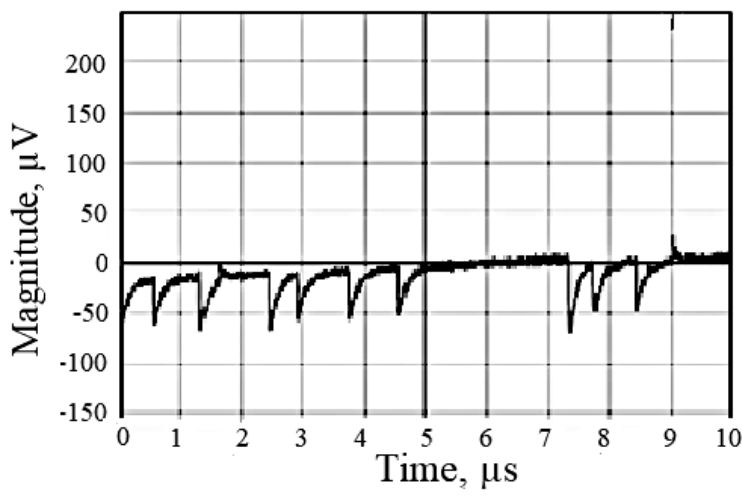

Рис. 4. Импульсы Тричела в воздушном разрядном промежутке поверхность-игла, зарегистрированные с помощью антенны.

Fig. 4. Trichel pulses in the surface-needle air discharge gap registered using an antenna.

Было установлено, что скорость нарастания фронта ЧР и скорость спада в обоих случаях совпадала, хотя амплитуда импульсов Тричела больше амплитуды наиболее характерных ЧР при переменном напряжении. Частота следования наиболее характерных сигналов ЧР на отрицательной полуволне с увеличением амплитуды приложенного напряжения увеличивалась. Мегаомметром были определены средние значения сопротивлений дефектного 50 МОм и работоспособного 3500 МОм фарфорового изоляторов. Измерения проводились лабораторных условиях. Погрешность измерения достигала до $50 \%$ в зависимости от влажности воздуха. Пробивное напряжение, при котором срабатывала релейная защита высоковольтного блока, для дефектного изолятора варьировалась от 12 кВ до 15 кВ. Исходя из соотношения сопротивлений работоспособного и дефектного фарфорового изоляторов была сделана оценка среднего пробивного напряжения работоспособного изолятора: 800 кВ. На практике такое большое пробивное напряжение создать невозможно, а это важно для обслуживания изоляторов линий электропередачи. Пробивное напряжение работоспособного фарфорового изолятора было оценено по динамике характеристик ЧР. При этом было выяснено, что в предпробойной ситуации ЧР следуют упорядоченно, спектр ЧР смещается из ВЧ в УВЧ область ЭМИ, а относительное количество наиболее характерных ЧР для дефектного изолятора, упорядочение которых наблюдается в предпробойной ситуации, растет в геометрической прогрессии кратно величине приложенного напряжения, что позволило оценить величину пробивного напряжения для бездефектного изолятора. В первую очередь такая зависимость связана с нарастанием тока утечки через изолятор, изучение структуры которого дает информацию о характеристиках ЧР, что в свою очередь позволяет предсказать предпробойную ситуацию.

Исследования статистических распределений ЧР для работоспособного фарфорового изолятора и ПЧР для работоспособного полимерного изолятора позволило сделать вывод, что ПЧР можно выделить на фоне внутренних ЧР. При этом ПЧР обусловлены увлажнением поверхности изоляторов и заметны только для полимерного изолятора (рис. 5), что позволяет различать наличие увлажненных опорных полимерных изоляторов при наличии фарфоровых изоляторов тарельчатого типа, причем на фоне внутренних ЧР, что важно с точки зрения классификации дефектов.

Пик величиной 0,12 В появляется при существенном увлажнении поверхности полимерных изоляторов и не проявляется при увлажнении поверхности фарфоровых изоляторов, что позволяет эффективно определять сильно увлажненные ПКИ, из которых изготавливают концевые и соединительные муфты силовых кабелей. Учет степени увлажнения и загрязнения поверхности полимерных изоляторов должен способствовать уменьшению выхода из строя изоляторов, прежде всего за счет предотвращения образования дуговых разрядов, а также ЧР, пронизывающие изолятор изнутри и разрушающих его, о чем свидетельствует ряд зарубежных исследований. Были проведены исследования по локализации источника ЧР различными датчиками. На рис. 6 изображены осциллограммы ЧР при постоянном приложенном напряжении, зарегистрированные контактным датчиком и антенной в ситуации далекой от пробоя разрядного промежутка. 
Видно, что ЧР возникают в одно и то же время, независимо от регистрации ЧР контактным ВИТ, или антенной, что можно определить по времени начала нарастания переднего фронта импульсов.

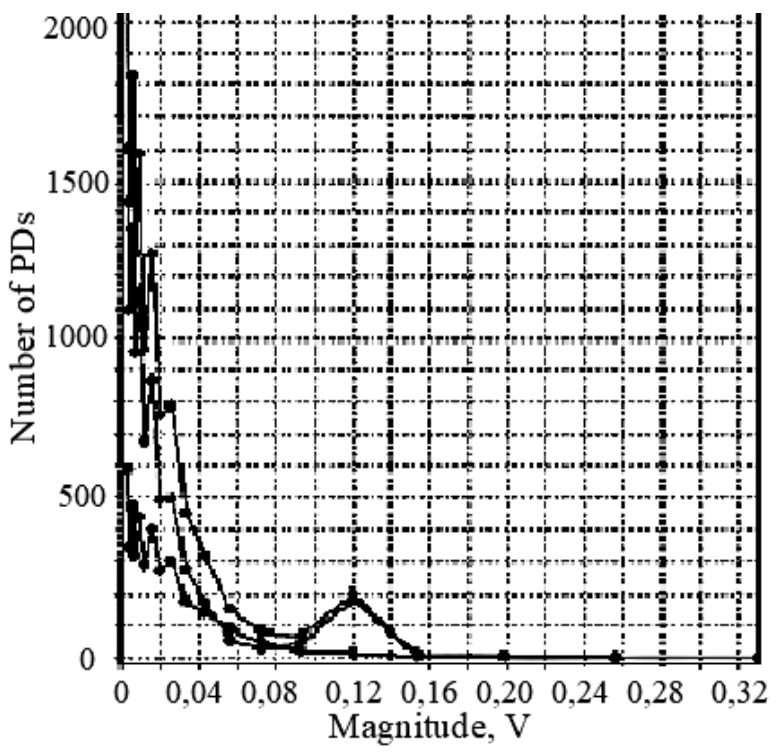

Рис. 5. Статистическое распределение ЧР типа 1 и типа 2. Увлажненный ПКИ, переменное напряжение 35 кВ. Снизу-вверх: ЧР с отрицательной, положительной полярностью и суммарное число импульсов. Развертка по горизонтали: амплитуда в вольтах (1 мВ=10 пКл). Масштаб по вертикали 100 ЧР на деление, масштаб по горизонтали 0,02 В/деление.

Fig. 5. Statistical distribution of PDs: type 1 and type 2. Humidified PCI, alternating voltage $35 \mathrm{kV}$. Bottom-up: PDs with negative, positive polarity and the total number of pulses. Horizontal sweep: amplitude in volts $(1 \mathrm{mV}=10 \mathrm{pC})$. Vertical scale 100 PD per division, horizontal scale $0.02 \mathrm{~V} / \mathrm{div}$.

По относительному смещению по времени нарастания сигналов 0,4 секунды, с учетом особенностей распространения ЧР: по воздуху для антенны и по ВЧ кабелю для ВИТ, было определено местоположение источника ЧР с точностью до 0,4 метра.

Исследование спектра ЧР работоспособного изолятора показало, что большинство ЧР располагаются в диапазоне до 90 МГц при условии, что приложенное напряжение далеко от пробивного (рис. 7).

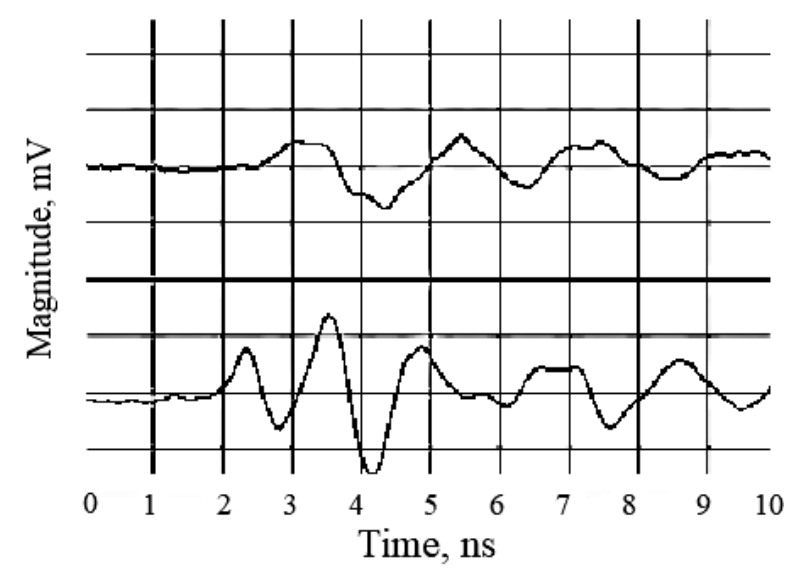

Рис. 6. Канал 1: ВИТ. Канал 2: антенна. Постоянное напряжение 8 кВ. Ситуация далекая от пробоя разрядного промежутка.

Fig. 6. Channel 1: VIT. Channel 2: antenna. The constant voltage is $8 \mathrm{kV}$. The situation is far from the breakdown of the discharge gap.

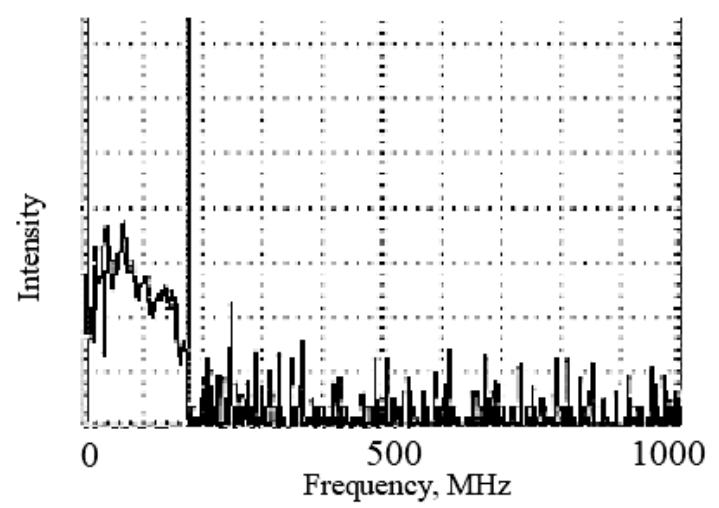

Рис. 7. Фурье-преобразование сигнала для работоспособного фарфорового изолятора. Переменное напряжение 10 кВ. Работоспособный изолятор.

Fig. 7. Fourier signal transformation for workable porcelain insulator. The alternating voltage is $10 \mathrm{kV}$.

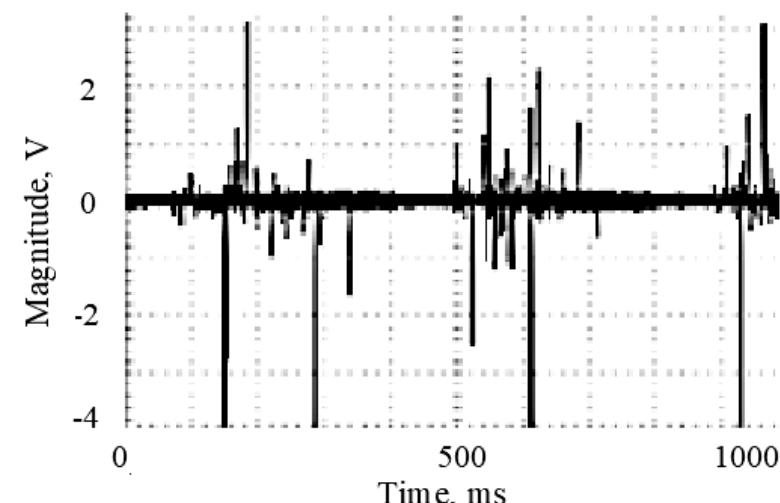

Рис. 8. ЭМД, настроенный на 434 МГц. Дефектный фарфоровый изолятор. Переменное напряжение 7 кB.

Fig. 8. $434 \mathrm{MHz}$ EMS. Defective porcelain insulator. Alternating voltage of $7 \mathrm{kV}$. 
Для работоспособного фарфорового изолятора пробивное напряжение очень велико, в отличие от дефектного изолятора аналогичного типа. При этом спектр ЧР смещается в УВЧ область ЭМИ, что является одним из признаков пробоя. Применение датчика УВЧ диапазона ЭМИ позволяет значительно увеличить дальность регистрации ЧР (рис. 8,9 ).

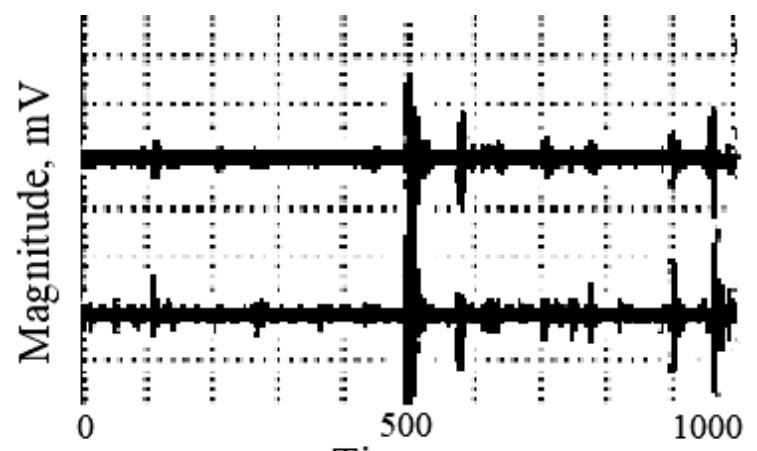

Time, ms

Рис. 9. ЭМД, настроенный на 434 МГц и ЭМД, настроенный на 1 ГГц. Работоспособный фарфоровый изолятор.

Fig. 9. EMS tuned to $434 \mathrm{MHz}$ and EMS tuned to $1 \mathrm{GHz}$. A workable porcelain insulator.

\section{IV. Выводы}

Установлено, что по характеристикам частичных разрядов с высокой степенью точности можно оценить пробивное напряжение фарфоровых изоляторов тарельчатого типа. Полученные данные коррелируют с результатами оценки пробивного напряжения, полученного путем сопоставления омического сопротивления дефектного и работоспособного фарфоровых изоляторов.

Предложен метод определения предпробойной ситуации модельного разрядного промежутка по характеристикам наиболее характерным ЧР вблизи нулевых значений приложенного переменного напряжения, которые в предпробойной ситуации следуют упорядоченно один за другим, что приводит к смещению спектра ЧР из ВЧ области в УВЧ область ЭМИ, что в свою очередь позволяет увеличить дальность регистрации ЧР и локализовать на значительном расстоянии дефектные изоляторы.

Для увлажненных полимерных изоляторов построены статистические распределения ЧР, на которых выделены ПЧР величиной 0,12 В не характерные для увлажненных аналогичным образом фарфоровых изоляторов тарельчатого типа.

Загрязнение и, как следствие, разрушение изолятора может стать причиной отключения потребителей от системы энергоснабжения. Известны случаи с более тяжелыми последствиями неудовлетворительного состояния изоляторов, когда отключение линии среднего напряжения приводило к крупным авариям, то есть отключениям линий более высокого напряжения. Предложенный способ учета степени увлажнения и загрязнения поверхности изоляторов по характеристикам ЧР позволит предотвратить аварийные ситуации в будущем.

Показано, что возможна локализация источника ЧР с высокой степенью точности (до 0,4 метра) при наличии одного источника ЧР, в нашем случае - это система электродов поверхность-игла и при условии, что применяются датчики ВЧ и УВЧ диапазона ЭМИ.

Полученные результаты могут быть полезными для внедрения в интеллектуальные системы энергоснабжения на основе искусственных нейронных сетей.

\section{БИБЛИОГРАФИЯ}

[1] Karimi M., Majidi M., Mirsaeedi H., Arefi M.M., Oskuoee M. A Novel Application of Deep Belief Networks in Learning Partial Discharge Patterns for Classifying Corona, Surface and Internal Discharges. IEEE Transactions On Industrial Electronics, 2019, vol. 1, no. 1, pp. 1049-1053. doi:10.1109/tie.2019.2908580.

[2] Majidi M., Fadali M. S., Etezadi-Amoli, M., Oskuoee M. Partial Discharge Pattern Recognition via Sparse Representation and ANN. IEEE Transactions on Dielectrics and Electrical Insulation, 2015, vol. 22, no. 2, pp. 1061-1070. doi:10.1109/tdei.2015.7076807.

[3] Tang J., Liu F., Zhang X., et al. Partial Discharge Recognition Based on SF6 Decomposition Products and Support Vector Machine. IET Sci. Meas. Technol., 2012, vol. 6, no. 1, pp. 198-204.

[4] Sahoo N.C., Salama M.M.A., Bartnikas R. Trends in Partial Discharge Pattern Classification: a Survey, IEEE Trans. Dielectr. Electr. Insul., 2005, vol. 12, no. 2, pp. 248-264.

[5] Sellars A.G., Farish O., Peterson M. UHF Detection of Leader Discharges in SF6, IEEE Trans. Dielectr. Electr. Insul., 1995, vol. 2, no. 1, pp. 143-154.

[6] Chen H.C. Partial Discharge Identification System for High Voltage Power Transformers Using Fractal Feature Based Extension Method, 
IET Sci. Meas. Technol., 2013, vol. 7, no. 2, pp. 77-84.

[7] Tang J., Jin M., Zeng F., Zhang X., Huang R. (2017). Assessment of PD Severity in GasInsulated Switchgear with an SSAE, IET Science, Measurement \& Technology, vol. 11, no. 4, pp. 423-430, doi:10.1049/iet-smt.2016.032.

[8] Jineeth J., Mallepally R., Sindhu T.K. Classification of Partial Discharge Sources in XLPE Cables by Artificial Neural Networks and Support Vector Machine. 2018 IEEE Electrical Insulation Conference (EIC), 2018, doi:10.1109/eic.2018.8481124.

[9] Chai. H., Phung. B.T., Mitchel. S., Application of UHF Sensors in Power System Equipment for Partial Discharge Detection: A Review. Sensors, vol. 19(5), no. 1029, doi:10.3390/s19051029.

[10]Zhang G., Zhang X., Cheng H., Tang, J., Ladder-Wise Calculation Method for $\mathrm{Z}$ Coordinate of Transformer PD Source Based on Planar Layout UHF Antenna Sensors. IEEJ Transactions on Electrical and Electronic Engineering, vol. 15, no. 3, pp. 340-345, doi:10.1002/tee.23061.

[11] Mondal M, Kumbhar G.B., Kulkarni S.V., Partialization of Partial Discharges inside a Transformer Winding Using a Ladder Network Constructed from Terminal Measurements. IEEE Transactions on Power Delivery, 2018, vol. 33, no. 3, pp. 1035-1043.

[12] Pan C., Tang J., Zhang Y., Luo X., Li X., Variation of Discharge Characteristics with Temperature in Moving Transformer Oil Contaminated by Metallic Particles, IEEE Access, 2018, pp. 40050-40058.

[13] Xiang C., Huang Z., Li J., Zhou Q., Yao W, Graphic Approaches for Faults Diagnosis for Camellia Insulating Liquid Filled Transformers
Based on Dissolved Gas Analysis, IEEE Transactions on Dielectrics and Electrical Insulation, 2018, vol. 25, no. 5, pp. 1897-1903.

[14] Kozioł M., Nagi. Ł., Kunicki. M., Urbaniec. I. (2019). Radiation in the Optical and UHF Range Emitted by Partial Discharges, Energies, 2019, vol. 12(22), no. 4334, doi:10.3390/en12224334.

[15] Kunicki. M.; Cichon. A. Application of a Phase Resolved Partial Discharge Pattern Analysis for Acoustic Emission Method in High Voltage Insulation Systems Diagnostics. Arch. Acoust., 2018, vol. 43, pp. 235-243.

[16] Wotzka D., Koziol M., Nagi L., Urbaniec I., Experimental Analysis of Acoustic Emission Signals Emitted by Surface Partial Discharges in Various Dielectric Liquids, 2nd International Conference on Dielectrics, ICD, Budapest, Hungary, 1-5 July 2018; pp. 1-5.

[17]Upton D., Saeed B., Mather P, Lazaridis P., Vieira M., Atkinson. R., Tachtatzi C., Wireless Sensor Network for Radiometric Detection and Assessment of Partial Discharge in High-Voltage Equipment, Radio Science, vol. 53, issue 3, 2018, pp. $357-364$.

[18] Markalous S.M., Tenbohlen S., $\quad$ Feser K., Detection and Location of Partial Discharges in Power Transformers Using Acoustic and Electromagnetic Signals, IEEE Trans. Dielectr. Electr. Insul., vol. 15, 2008, pp. 1576-1583.

[19] Arefifar S.A., Mohamed Y.A.R.I., El-Fouly T.H., Comprehensive Operational Planning Framework for Self-Healing Control Actions in Smart Distribution Grids, IEEE Trans. Power Syst, vol. 28, 2013, pp. 4192-4200.

[20] Wang D., Du L., Yao C., Yan J., UHF PD Measurement System with Scanning and Comparing Method, IEEE Trans. Dielectr. Electr. Insul, vol. 25, 2018, pp. 199-206.

\section{Сведения об авторах.}
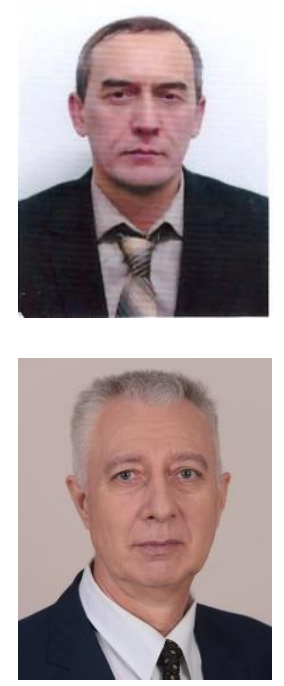

Гатауллин Айрат Мухамедович, доцент кафедры РЗА ФГБОУ ВО «КГЭУ». Область интересов: релейная защита, диагностика изоляторов высоковольтного электрооборудования методом частичных разрядов.

E-mail: gataullin1969@gmail.com

Губаев Дамир Фатыхович, профессор кафедры РЗА ФГБОУ ВО «КГЭУ». Область интересов: релейная защита, диагностика изоляторов высоковольтного электрооборудования методом частичных разрядов.

E-mail: $\underline{\text { d-gubaev@mail.ru }}$ 